\title{
CORRESPONDENCE Bronchiolitis severity is related to recurrent wheezing by age 3 years in a prospective, multicenter cohort
}

Pediatric Research (2020) 87:428-430; https://doi.org/10.1038/s41390019-0589-z

\section{INTRODUCTION}

Bronchiolitis is an important public health problem with a wide range of clinical severity and charges of $\sim \$ 1.7$ billion annually. ${ }^{1}$ Although most children with bronchiolitis are well enough to be cared for at home, $2-3 \%$ of U.S. children require hospitalization (i.e., severe bronchiolitis) annually. ${ }^{1}$ In addition to causing shortterm morbidity, bronchiolitis is also a risk factor for chronic respiratory morbidity (i.e., recurrent wheezing and childhood asthma). ${ }^{1}$ In 2009, Carroll et al. analyzed administrative data and reported a dose-response relationship between increasing severity of bronchiolitis (i.e., outpatient, emergency department, and hospitalization) and higher odds of developing childhood asthma. $^{2}$ Indeed, cohort studies estimate that $30-40 \%$ of infants with severe bronchiolitis will develop childhood asthma. ${ }^{3}$ It is unknown, however, if within the large group of children who require hospitalization ${ }^{1}$ there is an association between increased severity of illness and recurrent wheezing of childhood, and later asthma. Within this severe bronchiolitis population, one measure of severity is the peak intensity of support required (e.g., regular ward vs. intensive care). A second measure of severity is the duration of support needed on the regular ward (e.g., length-ofstay (LOS)). In this planned analysis of a prospective cohort of infants hospitalized for bronchiolitis, we hypothesized that both intensive care and extended severe illness without intensive care would be associated with recurrent wheezing by age 3 years.

\footnotetext{
METHODS

We analyzed prospective cohort data from the 35th Multicenter Airway Research Collaboration (MARC-35). The study design, setting, participants, and data collection have been described previously. ${ }^{4}$ During the 2011-2014 winter seasons, teams at 17 sites across 14 U.S. states consecutively enrolled infants hospitalized for bronchiolitis who met the American Academy of Pediatrics definition of bronchiolitis. ${ }^{5}$ Infants with heart-lung disease or a gestational age $<32$ weeks were excluded. After being found eligible through a brief screening interview, parents/ guardians completed a structured interview to assess patients' demographic characteristics, medical and environmental history, and to obtain detailed information regarding the present bronchiolitis episode. Further clinical data on the patients' evaluation, treatment and course were obtained via medical records. Nasopharyngeal aspirates (NPAs) were collected using a standardized procedure within $24 \mathrm{~h}$ of admission for each participant. Respiratory syncytial virus (RSV) types A and B were detected in NPAs using real-time reverse-transcriptase polymerase chain reaction. Follow-up is being conducted via parent telephone interviews biannually through age 6 years $(n=921$ participants in the longitudinal cohort) by the EMNet Coordinating Center at Massachusetts General Hospital. All sites obtained institutional
}

review board approval. Further details are provided in the Online Repository (Supplementary Methods).

The primary exposure, intensive care treatment, was defined as treatment in the intensive care unit (ICU), continuous positive airway pressure (CPAP), or intubation during the hospitalization. Since most children hospitalized with bronchiolitis do not require intensive care, our secondary exposure, extended severe illness without intensive care, utilized LOS in quartiles. Based on biannual parent phone interviews, the primary outcome, recurrent wheezing by age 3 years, was defined per the 2007 National Institutes of Health asthma guidelines ${ }^{6}$ as having $\geq 2$ corticosteroid-requiring exacerbations in 6 months or $\geq 4$ wheezing episodes in 1 year that last at least 1 day and affect sleep.

We calculated descriptive statistics including counts with percentages and medians with interquartile ranges (IQR). We assessed cumulative incidence of recurrent wheezing by intensive care treatment using a Kaplan-Meier plot. Analyses were performed using Stata 14.2 (StataCorp, College Station, TX). Twotailed $P<0.05$ was considered statistically significant.

We used multivariable Cox proportional hazards regression to estimate the association between intensive care treatment at baseline and development of recurrent wheezing by age 3 years. Factors were evaluated for inclusion in the model if they were found to be associated with the outcome in unadjusted analyses or were considered potentially clinically significant. We created a parsimonious model that excluded several variables that did not confound the main association (e.g., insurance status, smoke exposure, breastfeeding history, paternal history of asthma, and child history of eczema). For each covariate we evaluated the proportional hazards assumption required by the Cox model using Schoenfeld residuals. Since hazards were not proportional by age at enrollment, we applied age stratification to our Cox models. Based on Kaplan-Meier curves, we dichotomized age at enrollment as $<2$ months vs. $\geq 2$ months. Thus, the final regression model was stratified by age at enrollment and adjusted for sex, race/ethnicity, birth weight $<5 \mathrm{lbs}$, maternal history of asthma, ${ }^{7}$ parent history of eczema, viral etiology of bronchiolitis (RSV vs. non-RSV), and estimated median household income by home ZIP code at enrollment (Esri Business Analyst Desktop (Esri, Redlands, CA)). Because premature birth (3237 weeks), neonatal intensive care unit hospitalization at birth, previous breathing problems, and month of enrollment did not materially change the association between intensive care use and recurrent wheezing (data not shown), they were excluded from the multivariable model. Stratification by age of enrollment in months, rather than $<2$ vs. $\geq 2$ months, did not materially change the association between intensive care use and recurrent wheezing (data not shown). Moreover, high-flow oxygen use and antibiotic treatment also did not materially change the association between intensive care use and recurrent wheezing (data not shown) and therefore, were excluded from the multivariable model. Last, categorizing the viral etiology of the infant's bronchiolitis into different rhinovirus (RV) and RSV categories ${ }^{4}$ did not change the association between intensive care use and 


\begin{tabular}{|c|c|c|c|c|c|c|}
\hline Primary exposure & \multicolumn{3}{|c|}{ Recurrent wheezing by age 3 years $^{a}$} & \multicolumn{3}{|c|}{ Asthma by age 4 years $^{\mathrm{b}}$} \\
\hline Intensive care treatment (ICU, CPAP, and/or intubation) & 1.45 & $1.24-1.69$ & $<0.001$ & 1.62 & $1.02-2.59$ & 0.04 \\
\hline \multicolumn{7}{|l|}{ Characteristics at enrollment } \\
\hline Female & 0.90 & $0.73-1.10$ & 0.30 & 0.77 & $0.58-1.04$ & 0.09 \\
\hline \multicolumn{7}{|l|}{ Race/ethnicity } \\
\hline White non-Hispanic & (ref) & & & (ref) & & \\
\hline Black non-Hispanic & 0.89 & $0.68-1.15$ & 0.38 & 1.73 & $1.15-2.60$ & 0.009 \\
\hline Hispanic & 0.71 & $0.54-0.94$ & 0.02 & 1.43 & $0.96-2.14$ & 0.08 \\
\hline No & (ref) & & & (ref) & & \\
\hline Yes & 1.60 & $1.28-2.01$ & $<0.001$ & 2.19 & $1.58-3.03$ & $<0.001$ \\
\hline Unknown & 1.33 & $0.89-1.99$ & 0.17 & 2.24 & $1.30-3.87$ & 0.004 \\
\hline Non-RSV viral etiology & 2.01 & $1.55-2.60$ & $<0.001$ & 2.11 & $1.34-3.32$ & 0.001 \\
\hline \multicolumn{7}{|l|}{ Estimated median household income by ZIP code } \\
\hline$<\$ 40,000$ & 0.93 & $0.62-1.39$ & 0.71 & 0.77 & $0.44-1.37$ & 0.37 \\
\hline$\$ 40,000-\$ 79,999$ & 1.07 & $0.76-1.51$ & 0.72 & 0.92 & $0.64-1.31$ & 0.63 \\
\hline$\geq \$ 80,000$ & (ref) & & & (ref) & & \\
\hline
\end{tabular}

recurrent wheezing (data not shown). As a result, we have modeled viral etiology as RSV (including viral co-infections) vs. non-RSV. We accounted for potential clustering by site using a clustered sandwich estimator. Results are reported as hazard ratios (HRs) with $95 \%$ Cls.

For our secondary objective, we limited the analysis to children who did not receive intensive care treatment at baseline $(n=780)$. LOS was categorized into quartiles and modeled as a nominal categorical variable to avoid assuming a linear association. We used a Cox proportional hazards model stratified by age at enrollment, adjusted for the variables described above, and clustered by site, to estimate the association between extended severe illness and recurrent wheezing by age 3 years among children who did not receive intensive care treatment.

Wheezing in early childhood may be self-limited. ${ }^{8}$ Therefore, we also conducted a sensitivity analysis using asthma at age 4 years defined as parent report of doctor-diagnosed asthma by age 4 years and either asthma medication use (e.g., inhaled bronchodilator, inhaled corticosteroid, oral or systemic corticosteroid, montelukast) or asthma-related symptoms between 3.0 and 3.9 years. ${ }^{9}$ We used multivariable logistic regression to estimate the association between intensive care treatment at baseline and development of asthma by age 4 years. The logistic model was adjusted for the same variables used in the recurrent wheezing analysis. Results are reported as odds ratios (OR) with $95 \%$ Cls.

\section{RESULTS}

Among the 921 infants, 60\% were male and $44 \%$ non-Hispanic white (Supplementary Table S1). The median age at enrollment was 3.2 months (IQR 1.6-5.9 months) and 89\% had follow-up at 3 years. Recurrent wheezing by age 3 years was observed in 296 (32\%). Patients who received intensive care treatment tended to be younger. Compared to infants who did not require intensive care treatment, the risk of developing recurrent wheezing by age 3 years was significantly higher in those requiring intensive care $\left(P_{\text {log-rank }}=0.04\right.$ stratified by age at enrollment and site; Supplementary Fig. S1). In the multivariable Cox model, children undergoing intensive care treatment had a higher risk of developing recurrent wheezing by age 3 years (adjusted HR 1.45; 95\%Cl 1.24-1.69; $P<0.001$; Table 1). Intensive care treatment was similarly related to asthma at age 4 years (adjusted OR 1.62; $95 \% \mathrm{Cl} 1.02-2.59 ; P=0.04$; Table 1). Among the 780 (85\%) infants who did not receive intensive care, LOS in quartiles was not significantly associated with recurrent wheezing by age 3 years (Q4- vs. Q1-adjusted HR 1.29; 95\%Cl 0.79-2.09; $P=0.31$; Supplementary Table S2).

\section{DISCUSSION}

In this prospective, multicenter cohort of 921 infants hospitalized with bronchiolitis, infants who underwent intensive care during their hospitalization had significantly higher risk of recurrent wheezing by age 3 years and also, asthma at age 4 years. By contrast, children who did not require intensive care, but required long hospital stays were not at higher risk of recurrent wheezing. These prospective cohort results extend the dose-response relationship identified by Carroll et al. ${ }^{2}$ by demonstrating that within the large, high-risk population of infants who require hospitalization for bronchiolitis, a higher peak intensity of care (i.e., intensive care) is associated with childhood respiratory morbidity. 
One interpretation of these findings is that the association between the need for intensive care and recurrent wheezing by age 3 years is causal, since inflammation associated with severe respiratory infection has been related to long-term lung damage. ${ }^{10}$ Alternatively, intensive care use may be a marker of infants at risk for chronic respiratory morbidities. Specifically, children with pre-existing low lung function may be more likely to require intensive care and develop wheezing. ${ }^{10}$ Moreover, children who are genetically predisposed to severe infections may also be predisposed to wheezing. ${ }^{10}$ We are currently pursuing the underlying mechanisms of both severe bronchiolitis and postbronchiolitis respiratory morbidity. Meanwhile, given the association between ICU care and asthma at age 4 years, infants with bronchiolitis who require intensive care may need even closer post-discharge follow-up for wheezing, and note that this wheezing may eventually prove to be responsive to asthmadirected therapies.

This analysis has potential limitations. First, there is institutional variability in the use of intensive care measures. ${ }^{11}$ However, intensive care use for less ill infants would have biased the results towards the null. Second, recurrent wheezing may be transient, ${ }^{8}$ but the use of intensive care was also associated with an epidemiologic definition of asthma at age 4 years.

In summary, in a prospective, multicenter cohort of infants hospitalized with bronchiolitis, intensive care treatment was associated with respiratory morbidity during early childhoodrecurrent wheezing of childhood by age 3 years and also, asthma at age 4 years.

\section{ACKNOWLEDGEMENTS}

We thank the MARC-35 study hospitals and research personnel for their ongoing dedication to bronchiolitis and asthma research. We also thank Alkis Togias, MD for his many contributions to the study. This work was supported by the grants U01 Al087881, R01 Al-114552, R01 Al-108588, R01 Al-137091, and R01 Al-134940 from the National Institute of Allergy and Infectious Diseases and UG3/UH3 OD-023253 from the Office of the Director at the National Institutes of Health (Bethesda, MD). The content of this manuscript is solely the responsibility of the authors and does not necessarily represent the official views of the National Institutes of Health.

\section{AUTHOR CONTRIBUTIONS}

All authors made substantial contributions to conception and design, acquisition of data, or analysis and interpretation of data; drafting of the article or revising it critically for important intellectual content; and provided approval of the version to be published.

The MARC-35 Investigators:

Cindy S. Bauer ${ }^{3}$, Anne K. Beasley ${ }^{3}$, Markus Boos ${ }^{4}$, Juan Celedon ${ }^{5}$, Ari R. Cohen ${ }^{6}$, Michelle B. Dunn ${ }^{7}$, Michael R. Gomez ${ }^{8}$, Nancy R. Inhofe ${ }^{8}$, Sujit S. Iyer ${ }^{9}$, Federico R. Laham $^{10}$, Charles G. Macias ${ }^{11}$, Thida Ong ${ }^{4}$, Brian M. Pate ${ }^{12}$, Stephen C. Porter ${ }^{13}$, Henry T. Puls ${ }^{12}$, Wayne G. Shreffler ${ }^{6}$, Jonathan M. Spergel ${ }^{7}$, Michelle D. Stevenson ${ }^{14}$, Richard T. Strait ${ }^{13}$, Stephen J. Teach ${ }^{15}$, Amy D. Thompson ${ }^{16}$, Vincent J. Wang ${ }^{17}$, llana Y. Waynik $^{18}$, Susan $\mathrm{Wu}^{17}$

${ }^{3}$ Phoenix Children's Hospital, Phoenix, AZ, USA; ${ }^{4}$ Seattle Children's Hospital, Seattle, WA, USA; ${ }^{5}$ Children's Hospital of Pittsburgh, Pittsburgh, PA, USA; ${ }^{6}$ Massachusetts
General Hospital, Boston, MA, USA; ${ }^{7}$ Children's Hospital of Philadelphia, Philadelphia, PA, USA; ${ }^{8}$ Children's Hospital at St. Francis, Tulsa, OK, USA; ${ }^{9}$ Dell Children's Medical Center of Central Texas, Austin, TX, USA; ${ }^{10}$ Arnold Palmer Hospital for Children, Orlando, FL, USA; ${ }^{11}$ Texas Children's Hospital, Houston, TX, USA; ${ }^{12}$ The Children's Mercy Hospital \& Clinics, Kansas City, MO, USA; ${ }^{13}$ Cincinnati Children's Hospital and Medical Center, Cincinnati, OH, USA; ${ }^{14}$ Norton Children's Hospital, Louisville, KY, USA; ${ }^{15}$ Children's National Medical Center, Washington, DC, USA; ${ }^{16}$ Alfred I. duPont Hospital for Children, Wilmington, DE, USA; ${ }^{17}$ Children's Hospital of Los Angeles, Los Angeles, CA, USA and ${ }^{18}$ Connecticut Children's Medical Center, Hartford, CT, USA

\section{ADDITIONAL INFORMATION}

The online version of this article (https://doi.org/10.1038/s41390-019-0589-z) contains supplementary material, which is available to authorized users.

Competing interests: The authors declare no competing interests.

Publisher's note: Springer Nature remains neutral with regard to jurisdictional claims in published maps and institutional affiliations.

Jonathan M. Mansbach ${ }^{1}$, Kohei Hasegawa², Ruth J. Geller², Janice A. Espinola ${ }^{2}$, Ashley F. Sullivan ${ }^{2}$ and

Carlos A. Camargo $\mathrm{Jr}^{2}$ on behalf of the MARC-35 Investigators

${ }^{1}$ Department of Pediatrics, Boston Children's Hospital, Boston, MA, USA and ${ }^{2}$ Department of Emergency Medicine, Massachusetts General Hospital, Harvard Medical School, Boston, MA, USA Members of the MARC-35 Investigators are listed below the Acknowledgements Section Correspondence: Jonathan M. Mansbach (jonathan.mansbach@childrens.harvard.edu)

\section{REFERENCES}

1. Meissner, H. C. Viral bronchiolitis in children. N. Engl. J. Med. 374, 62-72 (2016).

2. Carroll, K. N. et al. The severity-dependent relationship of infant bronchiolitis on the risk and morbidity of early childhood asthma. J. Allergy Clin. Immunol. 123, 1055-1061 (2009).

3. Hasegawa, K., Mansbach, J. M. \& Camargo, C. A. Jr Infectious pathogens and bronchiolitis outcomes. Expert Rev. Anti Infect. Ther. 12, 817-828 (2014).

4. Hasegawa, K. et al. Association of nasopharyngeal microbiota profiles with bronchiolitis severity in infants hospitalised for bronchiolitis. Eur. Respir. J. 48, 1329-1339 (2016).

5. Ralston, S. L. et al. Clinical practice guideline: the diagnosis, management, and prevention of bronchiolitis. Pediatrics 134, e1474-e1502 (2014).

6. National Asthma Education and Prevention Program. Expert Panel Report 3 (EPR3): Guidelines for the Diagnosis and Management of Asthma-Summary Report 2007. J. Allergy. Clin. Immunol. 120, S94-S138. (2007)

7. Bacharier, L. B. et al. Determinants of asthma after severe respiratory syncytial virus bronchiolitis. J. Allergy Clin. Immunol. 130, 91-100 e103 (2012).

8. Martinez, F. D. Viral infections and the development of asthma. Am. J. Respir. Crit. Care Med. 151, 1644-1647 (1995). discussion 1647-1648.

9. Camargo, C. A. Jr. et al. Cord-blood 25-hydroxyvitamin D levels and risk of respiratory infection, wheezing, and asthma. Pediatrics 127, e180-e187 (2011).

10. Jackson, D. J., Gern, J. E. \& Lemanske, R. F. Jr Lessons learned from birth cohort studies conducted in diverse environments. J. Allergy Clin. Immunol. 139, 379-386 (2017).

11. Pierce, H. C. et al. Variability of intensive care management for children with bronchiolitis. Hosp. Pediatr. 5, 175-184 (2015). 